\title{
Ten-year analysis of saccular aneurysms in the Barrow Ruptured Aneurysm Trial
}

\author{
Robert F. Spetzler, MD, ${ }^{1}$ Cameron G. McDougall, MD, ${ }^{2}$ Joseph M. Zabramski, MD, ${ }^{1}$ \\ Felipe C. Albuquerque, MD, ${ }^{1}$ Nancy K. Hills, PhD, ${ }^{3,4}$ Peter Nakaji, MD, ${ }^{1}$ John P. Karis, MD, ${ }^{5}$ and \\ Robert C. Wallace, MD ${ }^{5}$
}

Departments of ${ }^{1}$ Neurosurgery and ${ }^{5}$ Neuroradiology, Barrow Neurological Institute, St. Joseph's Hospital and Medical Center, Phoenix, Arizona; ${ }^{2}$ Swedish Neuroscience Institute, Seattle, Washington; and Departments of ${ }^{3}$ Neurology and ${ }^{4}$ Epidemiology and Biostatistics, University of California, San Francisco, California

OBJECTIVE The authors present the 10-year results of the Barrow Ruptured Aneurysm Trial (BRAT) for saccular aneurysms. The 1-, 3-, and 6-year results of the trial have been previously reported, as have the 6-year results with respect to saccular aneurysms. This final report comparing the safety and efficacy of clipping versus coiling is limited to an analysis of those patients presenting with subarachnoid hemorrhage (SAH) from a ruptured saccular aneurysm.

METHODS In the study, 362 patients had saccular aneurysms and were randomized equally to the clipping and the coiling cohorts (181 each). The primary outcome analysis was based on the assigned treatment group; poor outcome was defined as a modified Rankin Scale (mRS) score $>2$ and was independently adjudicated. The extent of aneurysm obliteration was adjudicated by a nontreating neuroradiologist.

RESULTS There was no statistically significant difference in poor outcome (mRS score > 2) or deaths between these 2 treatment arms during the 10 years of follow-up. Of 178 clip-assigned patients with saccular aneurysms, $1(<1 \%)$ was crossed over to coiling, and $64(36 \%)$ of the 178 coil-assigned patients were crossed over to clipping. After the initial hospitalization, 2 of $241(0.8 \%)$ clipped saccular aneurysms and 23 of $115(20 \%)$ coiled saccular aneurysms required retreatment $(p<0.001)$. At the 10 -year follow-up, 93\% (50/54) of the clipped aneurysms were completely obliterated, compared with only $22 \%(5 / 23)$ of the coiled aneurysms $(p<0.001)$. Two patients had documented rebleeding, both died, and both were in the assigned and treated coiled cohort (2/83); no patient in the clipped cohort $(0 / 175)$ died $(p=0.04)$. In 1 of these 2 patients, the hemorrhage was not from the target aneurysm but from an incidental basilar artery aneurysm, which was coiled at the same time.

CONCLUSIONS There was no significant difference in clinical outcomes between the 2 assigned treatment groups as measured by $\mathrm{mRS}$ outcomes or deaths. Clinical outcomes in the patients with posterior circulation aneurysms were better in the coiling group at 1 year, but after 1 year this difference was no longer statistically significant. Rates of complete aneurysm obliteration and rates of retreatment favored patients who actually underwent clipping compared with those who underwent coiling.

Clinical trial registration no.: NCT01593267 (clinicaltrials.gov)

https://thejns.org/doi/abs/10.3171/2018.8.JNS181846

KEYWORDS Barrow Ruptured Aneurysm Trial; clip occlusion; coil embolization; intracranial aneurysm; ISAT; International Subarachnoid Aneurysm Trial; randomized trial; saccular aneurysms; SAH; subarachnoid hemorrhage; vascular disorders

$\mathrm{T}$ He 1-, 3-, and 6-year results of the Barrow Ruptured Aneurysm Trial (BRAT) have been previously reported., ${ }^{7,16,17}$ The 6-year results for saccular aneurysms have also been previously reported..$^{18}$ In this work we are presenting the 10-year final follow-up of saccular aneurysms.
Because the BRAT used a prospective intent-to-treat design that randomized all patients with nontraumatic subarachnoid hemorrhage (SAH) to either coiling or clipping on the basis of initial CT findings, enrollment included patients with angiographically negative $\mathrm{SAH}$, as well as

ABBREVIATIONS BRAT = Barrow Ruptured Aneurysm Trial; ISAT = International Subarachnoid Aneurysm Trial; $m$ RS = modified Rankin Scale; SAH = subarachnoid hemorrhage.

ACCOMPANYING EDITORIALS AND RESPONSE See pp 760-770. DOIs: 10.3171/2018.10.JNS182443, 10.3171/2018.10.JNS182444, 10.3171/2018.10.JNS182445; and 10.3171/2018.12.JNS182853.

SUBMITTED June 26, 2018. ACCEPTED August 21, 2018.

INCLUDE WHEN CITING Published online March 8, 2019; DOI: 10.3171/2018.8.JNS181846. 


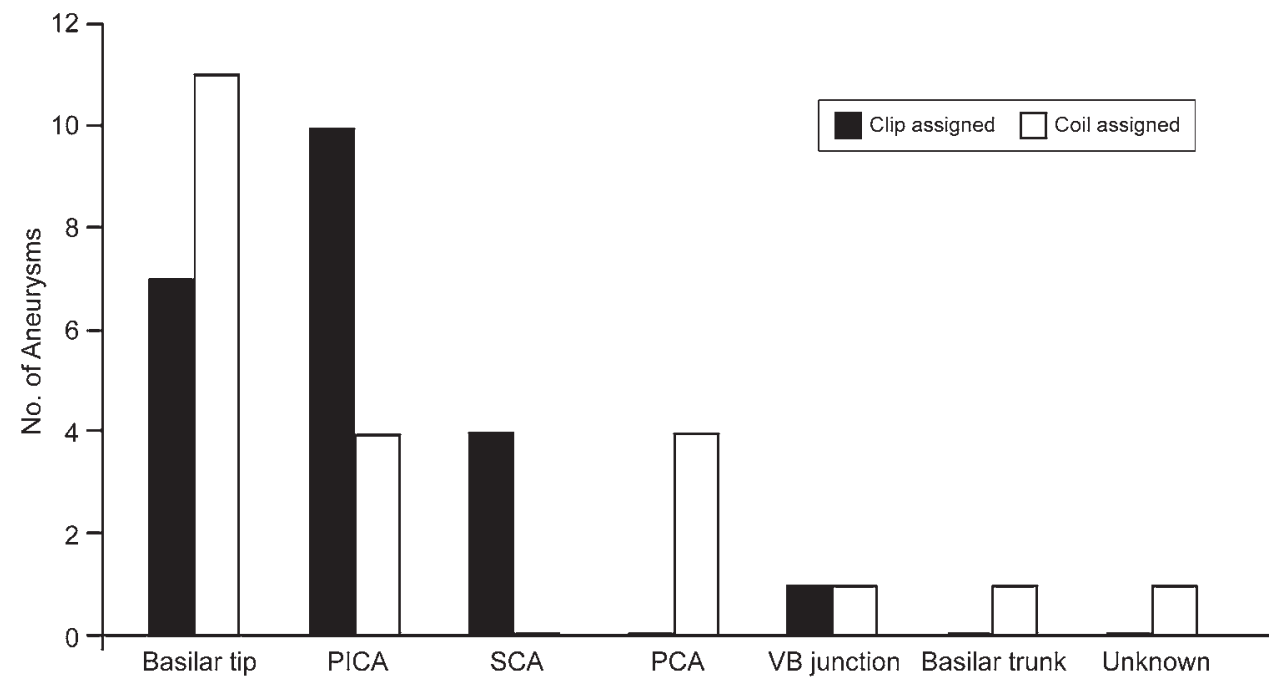

FIG. 1. Bar graph showing anatomical randomization of posterior circulation aneurysms. $P C A=$ posterior cerebral artery; $P I C A=$ posterior inferior cerebellar artery; SCA = superior cerebellar artery; $\mathrm{VB}=$ vertebrobasilar.

those with fusiform, blister, dissecting, and saccular aneurysms, and those with aneurysms associated with an arteriovenous malformation or fistula.

Although the BRAT was specifically initiated to be compared with the International Subarachnoid Aneurysm Trial (ISAT), we did not predetermine in our protocol that we were limiting the analysis to saccular aneurysms. However, given that every other randomized trial comparing clipping and coiling has been limited to saccular aneurysms, an analysis of this cohort in BRAT is appropriate.

\section{Methods}

The study protocol for the BRAT was approved by the institutional review board of St. Joseph's Hospital and Medical Center, Phoenix, Arizona, on November 12, 2002 , and is registered at ClinicalTrials.gov (NCT01593267). A detailed description of the study protocol was previously published. ' Briefly, all eligible patients with nontraumatic SAH who were admitted to the hospital between March 2003 and January 2007 were included. Of the 500 eligible patients, 29 were excluded ( 28 because consent was obtained in error and 1 because consent was rescinded), leaving 471 patients, of whom 238 were assigned to the intent-to-clip cohort and 233 to the intent-to-coil cohort.

Because there were no anatomical exclusion criteria, 6 categories of nontraumatic $\mathrm{SAH}$ were recorded: 1) nonaneurysmal SAHs, 2) aneurysms associated with arteriovenous malformations or fistulas, 3) fusiform aneurysms, 4) blister aneurysms, 5) dissecting aneurysms, and 6) saccular aneurysms.

Fifty-seven patients had a nonaneurysmal SAH. Although our 1-year outcome data included these untreated patients, they were excluded from further analysis in the 3 -year and 6-year publications because these outcomes were not associated with any treatment decision, and the outcomes in this nontreated group were identical between the 2 assigned cohorts. ${ }^{16}$ Of the 414 remaining patients, 212 were randomized to clipping and 202 to coiling. After a patient was assigned to coiling or clipping, the treating surgeon determined whether the assigned treatment was appropriate or whether the patient was best served by crossing over to the other treatment arm.

Aneurysm type was determined from angiographic studies, surgical inspection, and endovascular treatment. Because differences in aneurysm type are not always clear or well defined, as part of this post hoc analysis all nonsaccular aneurysms were rereviewed by a nontreating physician to assure the highest fidelity. Although some aneurysms were reclassified on review, statistical analysis revealed no significant difference between the initial and final adjudication. The final designation of aneurysm type for each lesion was used for this study. Saccular aneurysms represented $77 \%$ of all nontraumatic SAHs and $87 \%$ of all patients who received a diagnosis of an actual aneurysm. This report analyzes the outcomes of the 362 patients with saccular aneurysms.

\section{Outcome Analysis}

Follow-up data were collected at 6 months and at 1, 3, and 6 years and were collected for the current 10 -year final follow-up report. The modified Rankin Scale (mRS) assessments were performed by a qualified research nurse independent of the treating physician. Adverse events were recorded as they occurred. Outcomes were analyzed on an intent-to-treat basis by the assigned treatment group. The 2 groups were well matched, with the exception of aneurysm location in the posterior circulation (Figs. 1 and 2). ${ }^{16}$ Additional data recorded included aneurysm type, outcome based on actual treatment, outcome of crossover patients, rebleeding, death, aneurysm size, aneurysm location, and extent of aneurysm obliteration as assessed on imaging studies by a neuroradiologist not involved in treatment (R.C.W. or J.K.).

\section{Results}

Saccular aneurysms accounted for $76.8 \%$ (362/471) of all nontraumatic SAHs and $87.4 \%(362 / 414)$ of all patients 


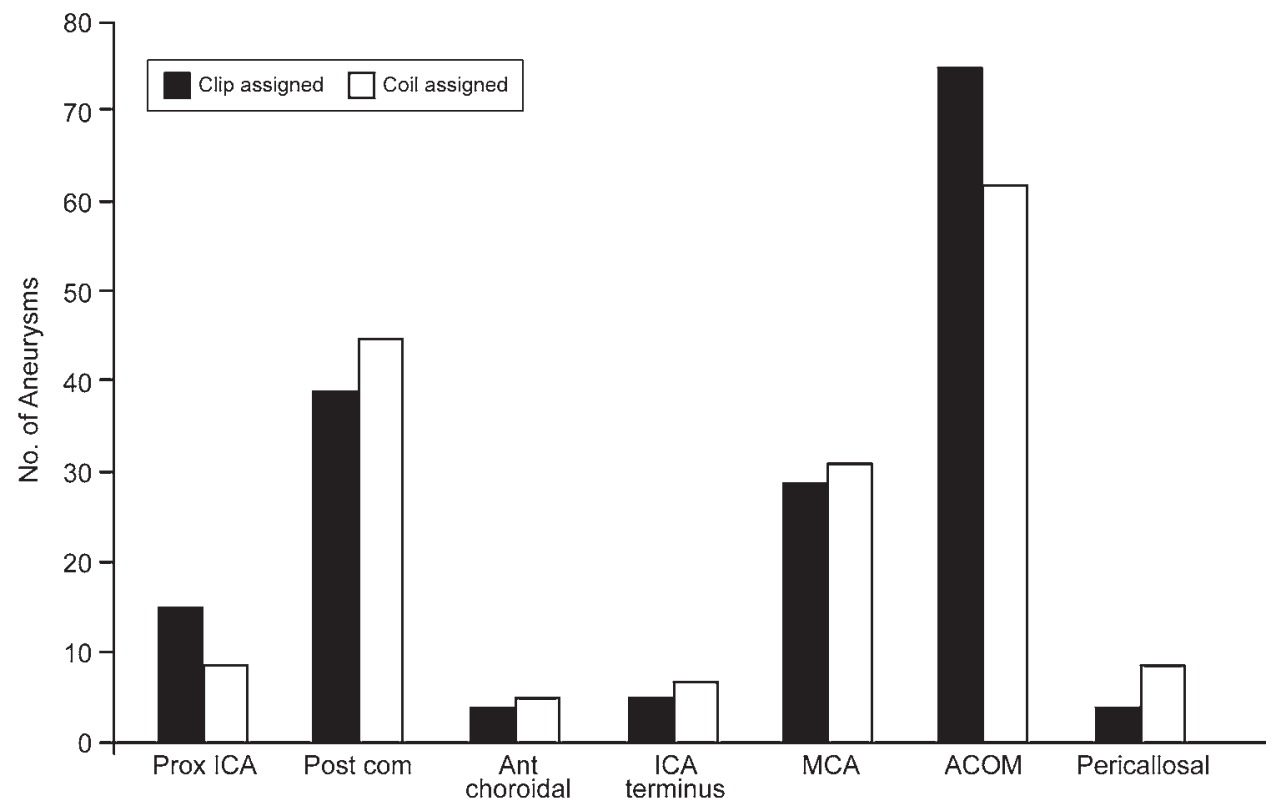

FIG. 2. Bar graph showing anatomical randomization of anterior circulation aneurysms. $A C O M=$ anterior communicating artery; Ant = anterior; ICA = internal carotid artery; MCA = middle cerebral artery; Post com = posterior communicating artery; Prox = proximal.

who had aneurysms. For the 362 patients with saccular aneurysms enrolled in the BRAT, randomization resulted in equal assignments of 181 patients to each cohort and good parity of Hunt and Hess scores. In each cohort, 3 patients died before treatment, leaving 178 for treatment. Of the 178 clip-assigned patients with saccular aneurysms, $1(<1 \%)$ was crossed over to the coiling arm, and $64(36 \%)$ of the 178 coil-assigned patients were crossed over to clipping arm.

\section{Intent-to-Treat Analysis}

For patients with saccular aneurysms, there was no sig- nificant difference in mRS scores $>2$ (Table 1) or deaths (Table 2) at any time during follow-up-from hospital discharge to 10 years posttreatment.

\section{Sentinel Events}

SAH and Deaths

There were 2 SAHs resulting in 2 deaths during the course of the study, at 7.5 and 9.5 years after treatment. Both patients were randomized to endovascular coiling and received that treatment (2/83). In 1 patient, the SAH was not from the target aneurysm but an incidental basilar artery aneurysm that was coiled at the time of the original

TABLE 1. Patients with saccular aneurysms with an $m R S$ score $>2$, grouped by assigned treatment

\begin{tabular}{|c|c|c|c|c|c|c|c|c|}
\hline \multirow[b]{2}{*}{ Time of FU } & \multirow{2}{*}{$\begin{array}{c}\text { Available } \\
\text { for Analysis }\end{array}$} & \multicolumn{3}{|c|}{ Coil-Assigned $(n=181)$} & \multicolumn{3}{|c|}{ Clip-Assigned $(n=181)$} & \multirow[b]{2}{*}{ p Value } \\
\hline & & $m R S>2$ & No. & $\%$ & $m R S>2$ & No. & $\%$ & \\
\hline Randomization & 362 & & & & & & & \\
\hline Discharge & 360 & 119 & 180 & 66.1 & 122 & 180 & 67.8 & 0.74 \\
\hline 6 mos & 306 & 42 & 159 & 26.4 & 49 & 147 & 33.3 & 0.19 \\
\hline $1 \mathrm{yr}$ & 317 & 43 & 158 & 27.2 & 52 & 159 & 32.7 & 0.29 \\
\hline 3 yrs & 307 & 52 & 154 & 33.8 & 50 & 153 & 32.7 & 0.84 \\
\hline 3 yrs w/ CF† & 320 & 52 & 159 & 32.7 & 50 & 161 & 31.1 & 0.75 \\
\hline 6 yrs & 297 & 56 & 148 & 37.8 & 59 & 149 & 39.6 & 0.76 \\
\hline 6 yrs w/ CF & 323 & 59 & 161 & 36.6 & 59 & 162 & 36.4 & 0.97 \\
\hline $10 \mathrm{yrs}$ & 264 & 70 & 131 & 53.4 & 68 & 133 & 51.1 & 0.71 \\
\hline 10 yrs w/ CF§ & 327 & 76 & 163 & 46.6 & 73 & 164 & 44.5 & 0.70 \\
\hline \multicolumn{9}{|c|}{$\begin{array}{l}\text { CF = carry forward; FU = follow-up. } \\
\text { * } p \text { values were calculated using the chi-square test. } \\
\text { t Includes patients seen at the } 1 \text {-year follow-up but not at the } 3 \text {-year follow-up. } \\
\text { I Includes patients seen at the } 1 \text { - or 3-year follow-up but not at the } 6 \text {-year follow-up. } \\
\text { § Includes patients seen at the 1-, 3-, or 6-year follow-up but not at the 10-year follow-up; it does not include patients no longer in the study or } \\
\text { those who could not be contacted at the 1-, 3-, 6-, and 10-year follow-ups. }\end{array}$} \\
\hline
\end{tabular}


TABLE 2. Saccular aneurysm deaths by assigned treatment group

\begin{tabular}{|c|c|c|c|c|c|c|c|c|}
\hline \multirow[b]{2}{*}{ Time of FU } & \multirow{2}{*}{$\begin{array}{c}\text { Available } \\
\text { for Analysis }\end{array}$} & \multicolumn{3}{|c|}{ Coil-Assigned ( $n=181$ ); No. of Deaths } & \multicolumn{3}{|c|}{ Clip-Assigned ( $n=181) ;$ No. of Deaths } & \multirow{2}{*}{$\begin{array}{c}p \\
\text { Value* }\end{array}$} \\
\hline & & Died & No. & $\%$ & Died & No. & $\%$ & \\
\hline $6 \mathrm{mos}$ & 306 & 18 & 159 & 11.3 & 19 & 147 & 12.9 & 0.67 \\
\hline $1 \mathrm{yr}$ & 317 & 19 & 158 & 12.0 & 23 & 159 & 14.5 & 0.52 \\
\hline 3 yrs & 307 & 24 & 154 & 15.6 & 27 & 153 & 17.6 & 0.63 \\
\hline 3 yrs w/ CF† & 320 & 24 & 159 & 15.1 & 27 & 161 & 16.8 & 0.68 \\
\hline 6 yrs & 297 & 28 & 148 & 18.9 & 31 & 149 & 20.8 & 0.68 \\
\hline 6 yrs w/ CF & 323 & 28 & 161 & 17.4 & 31 & 162 & 19.1 & 0.69 \\
\hline $10 \mathrm{yrs}$ & 264 & 43 & 131 & 32.8 & 46 & 133 & 34.6 & 0.76 \\
\hline 10 yrs w/ CF $\S$ & 327 & 43 & 163 & 26.4 & 46 & 164 & 28.0 & 0.74 \\
\hline
\end{tabular}

* $p$ values were calculated using the chi-square test.

† Includes patients seen at the 1-year follow-up but not at the 3-year follow-up.

$\ddagger$ Includes patients seen at the 1- or 3-year follow-up but not at the 6-year follow-up.

$\S$ Includes patients seen at the 1-, 3-, or 6-year follow-up but not at the 10-year follow-up; it does not include patients no longer in the study or those who could not be contacted at the 1-, 3-, 6-, and 10-year follow-ups.

SAH. There was no SAH during the 10-year follow-up in the clipped cohort $0 / 175(\mathrm{p}=0.04)$.

\section{Retreatments}

Aneurysm recurrence that required retreatment after discharge from hospital occurred in $<1 \%(2 / 241)$ of the patients whose aneurysms were clipped and in $20 \%$ (23/115; 3 patients underwent recoiling twice) of the patients who received coil treatment $(\mathrm{p}<0.001)$.

\section{Aneurysm Obliteration}

Clipping resulted in sustained aneurysm obliteration over time, whereas aneurysm obliteration continued to decrease over time in the patients who underwent coiling (Table 3).

\section{Analysis by Location \\ Posterior Circulation}

For the saccular aneurysms located in the posterior circulation, endovascular treatment was favored at 1 year $(p=0.03)$ but did not reach significance at any other recorded time (Table 4). There was no statistically significant difference in deaths at any follow-up time.

\section{Anterior Circulation}

For the anterior circulation aneurysms, there was good parity of all anatomical sites between the 2 assigned treatments (Fig. 2). At 10 years and at all other times, there was

TABLE 3. Complete aneurysm obliteration categorized by actual treatment

\begin{tabular}{lccc}
\hline \multicolumn{1}{c}{ Time of FU } & $\begin{array}{c}\text { Coiling No./Total } \\
(\%)\end{array}$ & $\begin{array}{c}\text { Clipping No./Total } \\
(\%)\end{array}$ & $\begin{array}{c}p \\
\text { Value }\end{array}$ \\
\hline Postop (baseline) & $60 / 115(52)$ & $205 / 236(87)$ & $<0.001$ \\
\hline 3 yrs & $26 / 58(45)$ & $103 / 117(88)$ & $<0.001$ \\
\hline 6 yrs & $16 / 40(40)$ & $95 / 100(95)$ & $<0.001$ \\
\hline 10 yrs & $5 / 23(22)$ & $50 / 54(93)$ & $<0.001$ \\
\hline
\end{tabular}

no significant difference in $\mathrm{mRS}$ scores $>2$ between the clipping and coiling groups (Table 5).

For anterior circulation aneurysms, deaths over the 10 years of the study were at parity by assigned treatment at all times. On average, at 10 years $34 \%$ of the patients in both assigned cohorts had died; when the cases carried forward were included, $27 \%$ of patients in each cohort had died.

\section{Discussion}

The BRAT was an all-inclusive trial in which randomization occurred for all patients with nontraumatic SAH before the source of the hemorrhage was determined. The high cross-over rate of $36 \%$ in the coiled cohort in the BRAT would probably be less if the study were repeated today, but it underscores the extent of selection bias involved in the ISAT enrollment process. Thus, the only aneurysms randomized in the BRAT that would have qualified for the ISAT are those that were randomized to coiling and then were actually treated by coiling. In addition, in the ISAT there were few posterior circulation aneurysms (only 2.7\%) and the average World Federation of Neurosurgical Societies grade and the Hunt and Hess grade was lower than in the BRAT. The all-inclusive design of the BRAT resulted in the inclusion of saccular, blister, fusiform, and dissecting aneurysms. Because all previous randomized trials ${ }^{5,6,8-11}$ have been restricted to saccular aneurysms, it is appropriate to analyze the BRAT for this same subgroup. This 10-year follow-up report provides the longest published follow-up of a cohort of nonselected patients with an SAH from a saccular aneurysm.

Among all the published prospective randomized trials of clipping versus coiling of saccular aneurysms that required equipoise for enrollment, only the ISAT claimed long-term benefit. ${ }^{5,6,9-11}$ The BRAT, like the ISAT, found a greater risk of subsequent SAH in the coiled cohort, probably from growth and recurrence of the aneurysm or coil compaction. ${ }^{3}$ In the ISAT, there was no requirement for postoperative documentation of aneurysms that were clipped, making it difficult to comment about the cause of the subsequent bleeds in that group. This lack of documentation has been a major criticism of the ISAT, and the high rebleeding rates in the clipped cohort are suspected 
TABLE 4. Patients with posterior circulation saccular aneurysms with an $m R S$ score $>2$ by assigned treatment

\begin{tabular}{|c|c|c|c|c|c|c|c|c|}
\hline \multirow[b]{2}{*}{ Time of FU } & \multirow{2}{*}{$\begin{array}{c}\text { Available } \\
\text { for Analysis }\end{array}$} & \multicolumn{3}{|c|}{ Coil-Assigned $(n=22)$} & \multicolumn{3}{|c|}{ Clip-Assigned $(n=22)$} & \multirow{2}{*}{$\begin{array}{c}p \\
\text { Value* }\end{array}$} \\
\hline & & $m R S>2$ & No. & $\%$ & $m R S>2$ & No. & $\%$ & \\
\hline $1 \mathrm{yr}$ & 40 & 6 & 21 & 29 & 12 & 19 & 63 & 0.03 \\
\hline $3 \mathrm{yrs}$ & 40 & 8 & 21 & 38 & 11 & 19 & 58 & 0.21 \\
\hline 3 yrs w/ CF† & 40 & 8 & 21 & 38 & 11 & 19 & 58 & 0.21 \\
\hline 6 yrs & 42 & 8 & 22 & 36 & 12 & 20 & 60 & 0.13 \\
\hline 6 yrs w/ CF $\ddagger$ & 42 & 8 & 22 & 36 & 12 & 20 & 60 & 0.13 \\
\hline $10 \mathrm{yrs}$ & 39 & 9 & 20 & 45 & 14 & 19 & 74 & 0.07 \\
\hline 10 yrs w/ CFß & 43 & 9 & 22 & 41 & 14 & 21 & 67 & 0.09 \\
\hline
\end{tabular}

to be at least partially due to the quality of the surgical care. ${ }^{1,4,12,14,15}$ Indeed, in a report documenting the rate of retreatment in the ISAT, ${ }^{2}$ it is noted that $30(3 \%)$ patients randomized to clipping had no clip applied during their initial craniotomy, and of these, 26 were subsequently coiled. This situation, in a group of small, mostly anterior circulation aneurysms where equipoise was a prerequisite before randomization, supports the allegation of inadequate surgical expertise in the ISAT. In contrast, the BRAT had no equipoise requirement, and only 1 patient was crossed over from clipping to coiling $(<1 \%)$. The fact that there was no $\mathrm{SAH}$ in the 10 years of follow-up of all clipped aneurysms after discharge in the BRAT lends credence to these concerns.

In the BRAT, the intent was treatment within the first 24 hours of admission, with the result that there was absolutely no difference in the pretreatment morbidity and mortality between the 2 randomized cohorts. In the ISAT, there was a 15-hour longer delay for clipping versus coiling after randomization, leading to an additional 11 rebleeding episodes and 12 more deaths in the clipping group than in the coiling group before treatment. These data again raise a question regarding the full commitment of all the participating physicians to the study. ${ }^{8}$

In the BRAT, in which the extent of aneurysm oblitera- tion was documented at all specified follow-up times and adjudicated by nontreating neuroradiologists, there was a significant difference $(\mathrm{p}<0.001)$ in the rate of complete aneurysm occlusion at 10 years that favored clipping over coiling; 93\% (50/54) versus 22\% (5/23) (Table 3). This difference explains the marked disparity noted in retreatment rates between the 2 study arms; $19 \%$ for the coiled cohort and $<1 \%$ for the clipped cohort ( $\mathrm{p}<0.001)$. The coiling retreatment rate was similar to that of the ISAT, in which $17.4 \%$ of coiled aneurysms were retreated. ${ }^{2}$ The high obliteration rate for the clipped cohort in the BRAT is the rational explanation for there being no recorded $\mathrm{SAH}$ in this cohort at 10 years $(0 / 175)$, versus the 2 SAHs that resulted in death in the coiled group $(2 / 83)(\mathrm{p}=0.04)$ (Tables $3-5)$. The fact that these fatal hemorrhages occurred at 7.5 and 9.5 years after the initial SAHs emphasizes the risk of aneurysm regrowth over time and the lack of value in reporting aggregate patient-years because the risk of rebleeding increases with time.

For the long-term ISAT follow-up, the title (The durability of endovascular coiling versus neurosurgical clipping of ruptured cerebral aneurysms: 18-year follow-up of the UK cohort of the International Subarachnoid Aneurysm Trial) suggests 18 years of follow-up, but the results are actually provided for only 10 years. The ISAT showed

TABLE 5. Patients with anterior circulation saccular aneurysms with an $\mathrm{mRS}$ score $>2$ by assigned treatment

\begin{tabular}{|c|c|c|c|c|c|c|c|c|}
\hline \multirow[b]{2}{*}{ Time of FU } & \multirow{2}{*}{$\begin{array}{c}\text { Available } \\
\text { for Analysis }\end{array}$} & \multicolumn{3}{|c|}{ Coil-Assigned $(n=161)$} & \multicolumn{3}{|c|}{ Clip-Assigned $(n=161)$} & \multirow{2}{*}{$\begin{array}{c}p \\
\text { Value }^{*}\end{array}$} \\
\hline & & $m R S>2$ & No. & $\%$ & $m R S>2$ & No. & $\%$ & \\
\hline $1 \mathrm{yr}$ & 277 & 37 & 137 & 27.0 & 40 & 140 & 28.6 & 0.77 \\
\hline 3 yrs & 267 & 44 & 133 & 33.1 & 39 & 134 & 29.1 & 0.48 \\
\hline 3 yrs w/ CF $\dagger$ & 280 & 44 & 138 & 31.9 & 39 & 142 & 27.5 & 0.42 \\
\hline $6 \mathrm{yrs}$ & 255 & 48 & 126 & 38.1 & 47 & 129 & 36.4 & 0.78 \\
\hline 6 yrs w/ CFf & 281 & 51 & 139 & 36.7 & 47 & 142 & 33.1 & 0.53 \\
\hline $10 \mathrm{yrs}$ & 225 & 61 & 111 & 55.0 & 54 & 114 & 47.4 & 0.26 \\
\hline 10 yrs w/ CF§ & 284 & 67 & 141 & 47.5 & 59 & 143 & 41.3 & 0.29 \\
\hline
\end{tabular}


that there was no difference in dependency between the clipped and coiled cohorts but that there were statistically significantly more deaths in the clipped cohort. ${ }^{9}$ This was not the case in the BRAT, in which, at the 10-year followup, the death rates in the anterior circulation were essentially the same in both cohorts.

\section{Study Limitations}

This review of the BRAT results for saccular aneurysms has several limitations. It is a post hoc analysis of a singlecenter study, and the designation of aneurysm types is subjective. Nevertheless, it provides long-term follow-up of a real-world, unselected population of patients with ruptured saccular aneurysms whose treatment assignment did not require equipoise.

\section{Conclusions}

There are a variety of opinions and conclusions reached by various experts resulting in the continued quest for the optimal treatment of patients with aneurysmal SAH. We are in agreement with the ISAT authors that a new trial is warranted. ${ }^{13}$ A prospective intent-to-treat trial is the only way to provide a credible answer as to which management method provides the better long-term result. In the BRAT, in patients with anterior circulation aneurysms, the clipping cohort had better outcomes than the coiling cohort for rebleeding, retreatment, and aneurysm obliteration, although there was no difference in clinical outcomes between the 2 groups.

\section{Acknowledgments}

Funding for this study was provided by the Barrow Neurological Foundation and the Hanley Aneurysm Fund. We thank the staff of Neuroscience Publications at Barrow Neurological Institute for assistance with manuscript preparation.

\section{References}

1. Batjer HH: ISAT study: is coiling better than clipping? Commentary. Surg Neurol 59:168-169, 2003

2. Campi A, Ramzi N, Molyneux AJ, Summers PE, Kerr RS, Sneade M, et al: Retreatment of ruptured cerebral aneurysms in patients randomized by coiling or clipping in the International Subarachnoid Aneurysm Trial (ISAT). Stroke 38:1538-1544, 2007

3. Hasan DM, Nadareyshvili AI, Hoppe AL, Mahaney KB, Kung DK, Raghavan ML: Cerebral aneurysm sac growth as the etiology of recurrence after successful coil embolization. Stroke 43:866-868, 2012

4. Kobayashi S: ISAT study: is coiling better than clipping? Commentary. Surg Neurol 59:167-168, 2003

5. Koivisto T, Vanninen R, Hurskainen H, Saari T, Hernesniemi J, Vapalahti M: Outcomes of early endovascular versus surgical treatment of ruptured cerebral aneurysms. A prospective randomized study. Stroke 31:2369-2377, 2000

6. Li ZQ, Wang QH, Chen G, Quan Z: Outcomes of endovascular coiling versus surgical clipping in the treatment of ruptured intracranial aneurysms. J Int Med Res 40:2145-2151, 2012

7. McDougall CG, Spetzler RF, Zabramski JM, Partovi S, Hills NK, Nakaji P, et al: The Barrow Ruptured Aneurysm Trial. J Neurosurg 116:135-144, 2012

8. Molyneux A, Kerr R, Stratton I, Sandercock P, Clarke M, Shrimpton J, et al: International Subarachnoid Aneurysm Trial (ISAT) of neurosurgical clipping versus endovascular coiling in 2143 patients with ruptured intracranial aneurysms: a randomised trial. Lancet 360:1267-1274, 2002

9. Molyneux AJ, Birks J, Clarke A, Sneade M, Kerr RS: The durability of endovascular coiling versus neurosurgical clipping of ruptured cerebral aneurysms: 18 year follow-up of the UK cohort of the International Subarachnoid Aneurysm Trial (ISAT). Lancet 385:691-697, 2015

10. Molyneux AJ, Kerr RS, Birks J, Ramzi N, Yarnold J, Sneade M, et al: Risk of recurrent subarachnoid haemorrhage, death, or dependence and standardised mortality ratios after clipping or coiling of an intracranial aneurysm in the International Subarachnoid Aneurysm Trial (ISAT): long-term follow-up. Lancet Neurol 8:427-433, 2009

11. Molyneux AJ, Kerr RS, Yu LM, Clarke M, Sneade M, Yarnold JA, et al: International subarachnoid aneurysm trial (ISAT) of neurosurgical clipping versus endovascular coiling in 2143 patients with ruptured intracranial aneurysms: a randomised comparison of effects on survival, dependency, seizures, rebleeding, subgroups, and aneurysm occlusion. Lancet 366:809-817, 2005

12. Raper DM, Allan R: International subarachnoid trial in the long run: critical evaluation of the long-term follow-up data from the ISAT trial of clipping vs coiling for ruptured intracranial aneurysms. Neurosurgery 66:1166-1169, 2010

13. Raymond J, Kotowski M, Darsaut TE, Molyneux AJ, Kerr RS: Ruptured aneurysms and the International Subarachnoid Aneurysm Trial (ISAT): What is known and what remains to be questioned. Neurochirurgie 58:103-114, 2012

14. Sade B, Mohr G: Critical appraisal of the International Subarachnoid Aneurysm Trial (ISAT). Neurol India 52:32-35, 2004

15. Solomon RA: ISAT study: is coiling better than clipping? Commentary. Surg Neurol 59:173, 2003

16. Spetzler RF, McDougall CG, Albuquerque FC, Zabramski JM, Hills NK, Partovi S, et al: The Barrow Ruptured Aneurysm Trial: 3-year results. J Neurosurg 119:146-157, 2013

17. Spetzler RF, McDougall CG, Zabramski JM, Albuquerque FC, Hills NK, Russin JJ, et al: The Barrow Ruptured Aneurysm Trial: 6-year results. J Neurosurg 123:609-617, 2015

18. Spetzler RF, Zabramski JM, McDougall CG, Albuquerque FC, Hills NK, Wallace RC, et al: Analysis of saccular aneurysms in the Barrow Ruptured Aneurysm Trial. J Neurosurg 128:120-125, 2018

\section{Disclosures}

Dr. McDougall is a consultant for Medtronic, plc, and Terumo Corp. Dr. Spetzler is a consultant for Carl Zeiss Meditec, AG, and receives royalties from Stryker Corp. and Kogent Surgical. Dr. Nakaji is a consultant for Carl Zeiss Meditec and a holder of GT Medical Technologies stock.

\section{Author Contributions}

Conception and design: Spetzler, Zabramski. Acquisition of data: Zabramski. Analysis and interpretation of data: Spetzler, Zabramski. Drafting the article: all authors. Critically revising the article: all authors. Reviewed submitted version of manuscript: all authors. Study supervision: Spetzler.

\section{Supplemental Information \\ Current Affiliations}

Dr. McDougall: Department of Neurosurgery, Johns Hopkins University School of Medicine, Baltimore, Maryland.

\section{Correspondence}

Robert F. Spetzler: c/o Neuroscience Publications, Barrow Neurological Institute, St. Joseph's Hospital and Medical Center, Phoenix, AZ.neuropub@barrowneuro.org. 